\title{
Doppler perfusion index in adolescents with non- alcoholic fatty liver disease
}

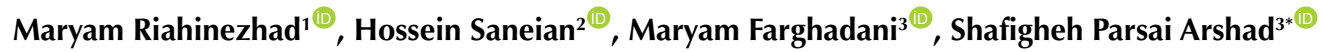 \\ 'Department of Radiology, Isfahan University of Medical Sciences, Isfahan, Iran \\ ${ }^{2}$ Pediatric Department, Child Growth and Development Research Center, Research Institute for Primordial Prevention of Non- \\ Communicable Disease, Isfahan University of Medical Sciences, Isfahan, Iran \\ ${ }^{3}$ Department of Radiology, School of Medicine, Isfahan University of Medical Sciences, Isfahan, Iran
}

*Correspondence to

Shafigheh Parsai Arshad, Email: sh.pa.1363@gmail. com; parsaiarshad@resident. mui.ac.ir

Received 25 February 2020 Accepted 9 June 2020 Published online 24 July 2020

Keywords: Doppler perfusion index, FibroScan, NonAlcoholic fatty liver disease, Steatosis

\begin{abstract}
Introduction: Non-alcoholic fatty liver disease (NAFLD) as a multi-factorial disorder is the most common cause of abnormal liver function tests in adolescents.

Objectives: The present study aimed to assess Doppler perfusion index (DPI) in adolescents with NAFLD as compared with healthy subjects.

Patients and Methods: Thirty-seven adolescents with NAFLD and 25 healthy individuals were enrolled in the study. Hemodynamic indices were measured using a color Doppler ultrasound machine. Severity of fatty liver disease and steatosis grade were assessed using FibroScan. The measured indices were peak systolic velocity (PSV), resistive index (RI), end-diastolic velocity (EDV), time-averaged mean velocity (TAMV), time-averaged peak velocity (TAPV), portal venous blood flow (PVBF) volume, hepatic arterial blood flow (HABF) volume, and DPI. Results: The mean of DPI in the case and control groups was $0.31 \pm 0.11$ and $0.31 \pm 0.09$, respectively $(P=0.972)$. HABF volume was significantly lower in the case group as compared with the control group (103.8 versus 153.3 respectively, $P=0.015)$. PSV, TAMV, mean velocity, and PVBF volume were significantly lower in the case group as compared with the control group $(P<0.05)$. Patients with a higher steatosis grade indicated a significantly lower mean of PSV and TAMV portal than controls $(P<0.05)$.

Conclusion: DPI was similar in adolescents with NAFLD and healthy subjects and was not associated with severity of fatty liver disease and steatosis grade. In addition, the percentage of steatosis would be increased with increased liver span.
\end{abstract}

Citation: Riahinezhad M, Saneian H, Farghadani M, Parsai Arshad S. Doppler perfusion index in adolescents with non-alcoholic fatty liver disease. Immunopathol Persa. 2021;7(1):e07. DOI:10.34172/ ipp.2021.07

\section{Introduction}

Non-alcoholic fatty liver disease (NAFLD) that has been regarded as the main cause of liver disease worldwide is defined as the accumulation of fat in the liver in the absence of excessive alcohol consumption or other detected liver pathologies (1). NAFLD is a multifactorial disorder closely associated with the metabolic syndrome and refers to a spectrum of diseases ranging from steatosis to steatohepatitis, fibrosis, and eventually cirrhosis (2). NAFLD is the major cause of liver transplantation in adults and is the most common cause of abnormal liver function tests in children and adolescents (3). Obesity and metabolic syndrome are the major risk factors of NAFLD in children $(4,5)$. The prevalence of NAFLD in developed countries has been estimated to be $20-30 \%$ in the general population $(6,7)$ with an estimated prevalence of $5-15 \%$ in children and adolescents (8). The increasing prevalence rates of childhood obesity and metabolic syndrome make

\begin{abstract}
Key point
The present study examined 37 adolescents with nonalcoholic fatty liver disease (NAFLD) and 25 healthy individuals and revealed that Doppler perfusion index (DPI) was not different between healthy adolescents and those with NAFLD. However, portal vein parameters based on ultrasound findings were found to be significantly lower in adolescents with NAFLD as compared with healthy subjects. In addition, patients with a higher steatosis grade showed a significantly lower mean of peak systolic velocity (PSV) and timeaveraged mean velocity (TAMV) of the main portal vein than healthy individuals.
\end{abstract}

NAFLD to be probably more common in children and adolescents which cause serious hepatic complications, and may even result in cirrhosis, portal hypertension, and death in adulthood (9). Therefore, there is an urgent need for non-invasive diagnostic methods for long-term monitoring in this regard.

There are currently no standardized criteria to diagnose fatty liver in children, since studies addressing the mentioned

\footnotetext{
Copyright (C) 2021 The Author(s); Published by Nickan Research Institute. This is an open-access article distributed under the terms of the Creative Commons Attribution License (http://creativecommons.org/licenses/by/4.0), which permits unrestricted use, distribution, and reproduction in any medium, provided the original work is properly cited.
} 
population have reported patterns of inflammation and fibrosis in the liver, which have been different from those observed in adults (10). Previous studies regarding fatty liver in children have shown that histology of fatty liver in children is also quite different from that of adults (11-13). Additionally, studies have indicated that the prevalence rate of fatty liver in adolescents is higher than that of children, which can be attributed to the hormonal changes at this age, lower physical activity and overweight $(14,15)$.

Various methods such as ultrasound, CT and MRI with different sensitivity and specificity have been used to check the grade of fatty liver. MRI is more accurate but is also a costly modality that cannot be performed at the bedside (16). Ultrasound is an economical method; however, patients' obesity affects its sensitivity and specificity (17).

FibroScan (transient elastography) as a rapid, noninvasive, and reproducible method has been recently developed for measuring liver stiffness. Studies focusing on histological findings and FibroScan results have revealed a relationship between the mentioned two diagnostic methods and have stated that many liver biopsies can be prevented in case of using this method $(18,19)$. However, another study has indicated that this method, due to different technicians' performance, can presents various results. Additionally, the results by FibroScan cannot be relied on to diagnose of advanced fibrosis in patients with fatty liver (20). Studies have revealed that the diagnostic function of FibroScan is acceptable for cirrhosis and in moderate fibrosis cases $(21,22)$. The benefits of this method include its ease of use, short duration, immediate outcome, high patient acceptance, and usability in outpatient clinics $(23,24)$. However, its use is limited to patients with ascites, individuals with morbid obesity, and/or patients with large amounts of fat in chest wall muscles (25).

Doppler ultrasound is another method that might be helpful in diagnosing fatty liver. Doppler perfusion index (DPI) that has been used in the detection of overt liver metastatic disease examines the relationship between HABF volume and total liver blood flow (26). Studies revealed that DPI has changed in patients with fatty liver $(16,27)$. As mentioned above, several methods with different weaknesses and strengths have been used to diagnose fatty liver in patients; however, there is limited evidence regarding the application of DPI as compared to other diagnostic modalities, especially in adolescence.

\section{Objectives}

The present study was carried out with the aim of comparing the application of DPI and FibroScan in the diagnosis of NAFLD in adolescents.

\section{Patients and Methods}

\section{Study design}

A total of 37 adolescents with NAFLD (confirmed by pediatric gastroenterologist) that referred to Imam Hossein hospital, Isfahan, Iran were recruited consecutively from
January to December 2018. Inclusion criteria for patients were the age range of 10-18 years, no history of liver surgery or liver mass, and lack of kidney or heart failure. In addition, 25 unrelated healthy individuals were recruited from the same hospital as the control group.

Collected data were age, gender, body mass index (BMI) that was measured as weight $(\mathrm{kg})$ divided by height $(\mathrm{m})$ squared, severity of fatty liver disease including mild, moderate, and severe, and steatosis severity including grades of S0, S1, S2, and S3 determined based on the extent of fat in the liver as follows; S0: steatosis of less than 5\%, S1; steatosis of $5 \%-33 \%$, S2; steatosis of $34 \%-66 \%$, and S3; steatosis of more than $66 \%$ (2).

Transient elastography was performed with FibroScan using the standard $\mathrm{M}$ probe. All FibroScan examinations were performed according to the manufacturer's specifications by a single trained operator blinded to DPI results. Liver stiffness measurements were performed on the right lobe through inter-costal spaces of patients lying in the dorsal decubitus position with the right arm in maximum abduction.

To measure hemodynamic indices and calculate DPI, a color Doppler ultrasound machine (Philips Affiniti 50) was used. The examination was performed after a 12hour fasting and drug-free period. The measurements were taken in a supine position. Portal vein measurements were taken right at the origin of the vessel from the splenic and superior mesenteric veins. Moreover, hepatic artery measurements were taken right at the origin of the vessel from the celiac axis. The Doppler waveforms were recorded over at least four cardiac cycles, and all patients were asked to briefly hold their breath during the procedure. The measured indices were peak systolic velocity (PSV), resistive index (RI), end-diastolic velocity (EDV), time-averaged mean velocity (TAMV), timeaveraged peak velocity (TAPV), portal venous blood flow (PVBF) volume, hepatic arterial blood flow (HABF) volume, and DPI (using the following formula; hepatic artery flow volume/portal artery flow volume + hepatic artery flow volume). All measurements were obtained at least three times for each patient, and the average values were regarded as Doppler findings.

\section{Ethics issues}

The research followed the Declaration of Helsinki principles. The Institutional Ethics Committee at Isfahan University of Medical Sciences approved all study protocols (IR.MUI.REC.1396.3.754). Accordingly, written informed consent was obtained from all subjects before any intervention. The present study was extracted from residential thesis of Shafigheh Parsai Arshad in the department of radiology at this university (Thesis\#396754).

\section{Data analysis}

The statistical analysis was performed using SPSS statistics, version 24.0 (SPSS, Chicago, IL, USA). Data are 
presented as means \pm SD and number (\%) for categorical and continuous variables, respectively. An independent samples $t$-test and a chi-square test were used to compare target variables between case and control groups. With regard to severity of fatty liver disease and steatosis grades, Kruskal-Wallis one-way analysis of variance was used to compare DPI and Doppler parameters between the case and control groups. In addition, Spearman's correlation coefficient was used to evaluate the relationship of DPI with liver span and steatosis percentage. In all statistical analysis, the significance level was considered to be less than 0.05 .

\section{Results}

The patients' demographics, severity of fatty liver disease, and steatosis grade are demonstrated in Table 1 . The mean age of case and control groups was similar $(P=0.073)$. In case group, $21.6 \%$ of patients were female whereas $48 \%$ of control group were female $(P=0.029)$. The mean of BMI in the case group was significantly higher than that of the control group $(P=0.001)$. Severity of fatty liver disease was mild for majority of the patients $(79.4 \%)$ and was moderate in $20.6 \%$ of cases. Steatosis grades in patients were; S0: seven patients, S1: three patients, S2: six patients, and S3: 21 patients.

Table 2 presents the comparison of Doppler parameters and DPI between the case and control groups. Maximum PSV, hepatic artery PSV, RI, EDV, mean velocity, TAMV, TAPV, and area of hepatic were not significantly different between the case and control groups $(P>0.05)$. HABF volume in the case group was significantly lower than that of the control group (103.8 versus 153.3 , respectively, $P$ $=0.015$ ). Of portal vein parameters, PSV, mean velocity, TAMV, and PVBF volume were significantly lower in

Table 1. Characteristics of studied patients

\begin{tabular}{lccc}
\hline Characteristics & Case group & Control group & $\boldsymbol{P}$ value \\
\hline Age (year) & $11.4 \pm 1.7$ & $12.4 \pm 2.3$ & 0.073 \\
Gender & & & \\
$\quad$ Male & $29(78.4)$ & $13(52.0)$ & 0.029 \\
$\quad$ Female & $8(21.6)$ & $12(48.0)$ & \\
BMl (kg/m $\left.{ }^{2}\right)$ & $27.8 \pm 4.6$ & $23.9 \pm 3.4$ & 0.0001 \\
Severity of fatty liver disease & & \\
Mild & $27(79.4)$ & - & \\
Moderate & $7(20.6)$ & - & \\
Steatosis & & & \\
S0 & $7(18.9)$ & - & \\
S1 & $3(8.1)$ & - & \\
S2 & $6(16.2)$ & - & \\
S3 & $21(56.8)$ & - & \\
\hline
\end{tabular}

Data are presented as means \pm SD or number $(\%)$. the case group as compared with the control group $(P<$ $0.05)$. Area of portal vein was similar between the case and control groups $(P=0.385)$. Moreover, the mean of DPI in the case group was similar to that of the control group ( 0.31 versus 0.31 respectively, $P=0.972$ ).

Table 3 shows the comparison of Doppler parameters and DPI between the controls and cases with respect to fatty liver disease and steatosis grade. All hepatic artery parameters were similar, and no significant differences were noted between patients with mild or moderate fatty liver disease and controls $(P>0.05)$. Of portal vein parameters, PSV, TAMV, and PVBF volume were significantly lower in patients with mild or moderate fatty liver disease as compared with controls $(P<0.05)$. The other portal vein parameters were not different between the two groups $(P>0.05)$. Additionally, only area of hepatic artery was significantly different between two groups $(P=0.039)$ and other hepatic artery parameters were not different between controls and patients considering their steatosis grade $(P<0.05)$. In addition, patients with regard to steatosis grade indicated a significantly lower mean of PSV portal and TAMV portal than control subjects $(P<0.05)$.

Figures 1 and 2 demonstrate the correlation of steatosis percentage with DPI and liver span, respectively. As shown, a weak, negative, and statistically non-significant correlation was observed between steatosis percentage and

Table 2. Doppler parameters and DPI in the case group versus control group

\begin{tabular}{llll}
\hline Parameters & Control group & Case group & P-value \\
\hline Parameters of hepatic artery & & & \\
\hline Maximum PSV & $19.2 \pm 5.5$ & $20.6 \pm 7.2$ & 0.421 \\
PSV hepatic $(\mathrm{cm} / \mathrm{s})$ & $47.4 \pm 22.6$ & $46.1 \pm 12.6$ & 0.774 \\
RI hepatic & $0.66 \pm 0.08$ & $0.69 \pm 0.10$ & 0.210 \\
EDV hepatic $(\mathrm{cm} / \mathrm{s})$ & $16.3 \pm 7.8$ & $13.5 \pm 5.1$ & 0.096 \\
Mean velocity hepatic & $15.5 \pm 8.0$ & $13.3 \pm 5$ & 0.232 \\
TAMV hepatic & $14.2 \pm 8.3$ & $12.9 \pm 5.3$ & 0.456 \\
TAPV hepatic & $28.7 \pm 13.1$ & $24.0 \pm 7.8$ & 0.093 \\
Area of hepatic & $0.16 \pm 0.07$ & $0.14 \pm 0.06$ & 0.355 \\
HABF volume & $153.3 \pm 101.5$ & $103.8 \pm 52.7$ & 0.015 \\
\hline Parameters of portal vein & & & \\
\hline PSV portal (cm/s) & $28.1 \pm 13.7$ & $19.7 \pm 4.1$ & 0.001 \\
Mean velocity portal & $17.0 \pm 9.2$ & $13.3 \pm 4.1$ & 0.041 \\
TAMV portal & $16.4 \pm 15.6$ & $9.5 \pm 3.6$ & 0.014 \\
Area of portal vein & $0.41 \pm 0.16$ & $0.44 \pm 0.17$ & 0.385 \\
PVBF volume & $325.7 \pm 174.6$ & $227.5 \pm 116.1$ & 0.010 \\
DPI & $0.31 \pm 0.11$ & $0.31 \pm 0.09$ & 0.972 \\
\hline Peak Svsolic Velocity & & & \\
\hline
\end{tabular}

Peak Systolic Velocity (PSV), Resistive Index (RI), End-Diastolic Velocity (EDV), time-averaged mean velocity (TAMV), time-averaged peak velocity (TAPV), Portal venous blood flow (PVBF) volume, Hepatic arterial blood flow (HABF)volume, Doppler Perfusion Index (DPI).

Data are presented as means \pm SD. $P$ values are calculated by an independent samples $t$ test. 
Table 3. Doppler parameters and DPI in controls versus patients with regard to fatty liver disease severity and steatosis grade

\begin{tabular}{|c|c|c|c|c|c|c|c|c|}
\hline \multirow{2}{*}{ Parameters } & \multicolumn{2}{|c|}{ Fatty liver disease } & \multirow{2}{*}{ Control } & \multirow{2}{*}{$P$ value } & \multicolumn{2}{|c|}{ Steatosis grade } & \multirow{2}{*}{ Control } & \multirow{2}{*}{$P$ value } \\
\hline & Mild & Moderate & & & S0 \& S1 & $\mathrm{S} 2 \& \mathrm{~S} 3$ & & \\
\hline \multicolumn{9}{|l|}{ Parameters of hepatic artery } \\
\hline Maximum PSV & $20.9 \pm 7.6$ & $19.1 \pm 5.6$ & $19.2 \pm 5.5$ & 0.580 & $20.5 \pm 9.0$ & $20.7 \pm 6.6$ & $19.2 \pm 5.5$ & 0.713 \\
\hline PSV hepatic (cm/s) & $46.1 \pm 13.1$ & $46.4 \pm 11.4$ & $47.4 \pm 22.6$ & 0.959 & $46.3 \pm 14.4$ & $46.1 \pm 12.1$ & $47.4 \pm 22.6$ & 0.810 \\
\hline RI hepatic & $0.69 \pm 0.11$ & $0.72 \pm 0.06$ & $0.66 \pm 0.08$ & 0.358 & $0.64 \pm 0.17$ & $0.71 \pm 0.06$ & $0.66 \pm 0.08$ & 0.068 \\
\hline EDV hepatic $(\mathrm{cm} / \mathrm{s})$ & $13.6 \pm 5.1$ & $13.0 \pm 5.4$ & $16.3 \pm 7.8$ & 0.245 & $14.9 \pm 6.8$ & $13.0 \pm 4.3$ & $16.3 \pm 7.8$ & 0.341 \\
\hline Mean velocity hepatic & $13.4 \pm 5.2$ & $13.2 \pm 4.5$ & $15.5 \pm 8.0$ & 0.492 & $14.5 \pm 6.5$ & $12.8 \pm 4.2$ & $15.5 \pm 8.0$ & 0.617 \\
\hline TAMV hepatic & $13.1 \pm 5.3$ & $11.8 \pm 5.6$ & $14.2 \pm 8.3$ & 0.694 & $16.2 \pm 5.6$ & $11.6 \pm 4.7$ & $14.2 \pm 8.3$ & 0.093 \\
\hline TAPV hepatic & $23.8 \pm 7.8$ & $25.1 \pm 8.4$ & $28.7 \pm 13.1$ & 0.239 & $26.2 \pm 8.6$ & $23.2 \pm 7.4$ & $28.7 \pm 13.1$ & 0.201 \\
\hline Area of hepatic & $0.14 \pm 0.05$ & $0.17 \pm 0.07$ & $0.16 \pm 0.07$ & 0.316 & $0.11 \pm 0.05$ & $0.16 \pm 0.06$ & $0.16 \pm 0.07$ & 0.039 \\
\hline HABF volume & $105.5 \pm 55.0$ & $96.3 \pm 44.4$ & $153.3 \pm 101.5$ & 0.050 & $111.1 \pm 49.3$ & $101.0 \pm 54.5$ & $153.3 \pm 101.5$ & 0.140 \\
\hline \multicolumn{9}{|l|}{ Parameters of portal vein } \\
\hline PSV Portal $(\mathrm{cm} / \mathrm{s})$ & $20.1 \pm 4.9$ & $18.1 \pm 3.6$ & $28.1 \pm 13.7$ & 0.004 & $20.0 \pm 2.3$ & $19.6 \pm 5.4$ & $28.1 \pm 13.7$ & 0.001 \\
\hline Mean velocity portal & $13.6 \pm 4.1$ & $11.7 \pm 3.8$ & $17.0 \pm 9.2$ & 0.105 & $14.6 \pm 4.0$ & $12.7 \pm 4.0$ & $17.0 \pm 9.2$ & 0.139 \\
\hline TAMV portal & $9.9 \pm 3.5$ & $8.1 \pm 3.6$ & $16.4 \pm 15.6$ & 0.047 & $11.1 \pm 4.4$ & $8.9 \pm 3.1$ & $16.4 \pm 15.6$ & 0.001 \\
\hline Area of portal & $0.42 \pm 0.14$ & $0.56 \pm 0.24$ & $0.41 \pm 0.16$ & 0.102 & $0.38 \pm 0.15$ & $0.47 \pm 0.18$ & $0.41 \pm 0.16$ & 0.260 \\
\hline PVBF volume & $243.4 \pm 119.5$ & $159.3 \pm 71.5$ & $325.7 \pm 174.6$ & 0.014 & $238.8 \pm 118.7$ & $223.3 \pm 117.1$ & $325.7 \pm 174.6$ & 0.056 \\
\hline DPI & $0.31 \pm 0.10$ & $0.35 \pm 0.06$ & $0.31 \pm 0.11$ & 0.580 & $0.32 \pm 0.08$ & $0.31 \pm 0.10$ & $0.31 \pm 0.11$ & 0.888 \\
\hline
\end{tabular}

PSV, peak systolic velocity; RI, resistive index; EDV, end-diastolic velocity; TAMV, time-averaged mean velocity, TAPV, time-averaged peak velocity; PVBF, Portal venous blood flow; HABF, Hepatic arterial blood flow; DPI, Doppler perfusion index.

Data are presented as means \pm SD. P-values are calculated by one-way ANOVA

DPI (Spearman's correlation coefficient $=-0.071, P=0.961$; Figure 1). However, a positive and statistically significant correlation was observed between steatosis percentage and liver span (Spearman's correlation coefficient = $0.527, P=0.001$; Figure 2). The mentioned findings reveal that the percentage of steatosis would be increased with increased liver span.

\section{Discussion}

In the present study, Doppler indices of hepatic and portal venous flow volume, steatosis grades, and disease severity calculated by FibroScan were evaluated in adolescents with

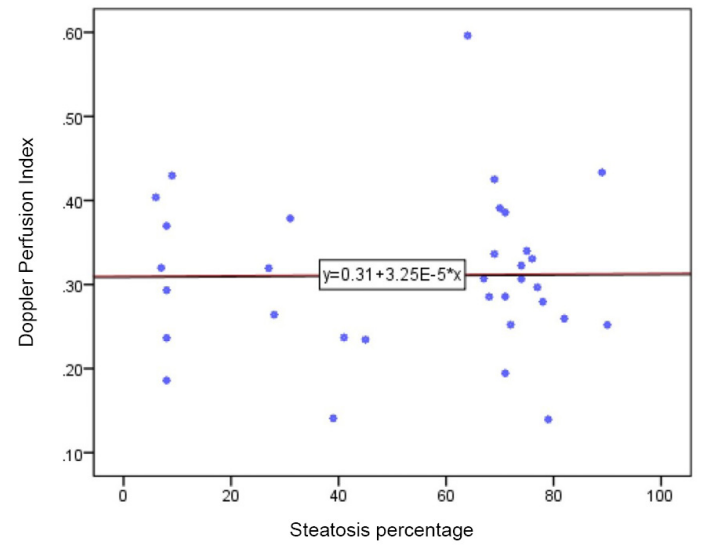

Figure 1. Correlation between Doppler Perfusion Index and steatosis percentage (Spearman's correlation coefficient $=-0.071, P$ value $=0.961$ )
NAFLD, and then the findings were compared with results of healthy subjects. The obtained findings revealed that portal vein parameters in adolescents with NAFLD were significantly lower than those of healthy subjects. DPI was similar in adolescents with or without NAFLD. Base on FibroScan results, DPI was not associated with steatosis grades and disease severity. The steatosis percentage in adolescents with NAFLD was significantly related to liver span, which means that patients with a larger liver span had a higher steatosis percentage.

DPI as the main focus of the present study was not significantly different between the case and control groups

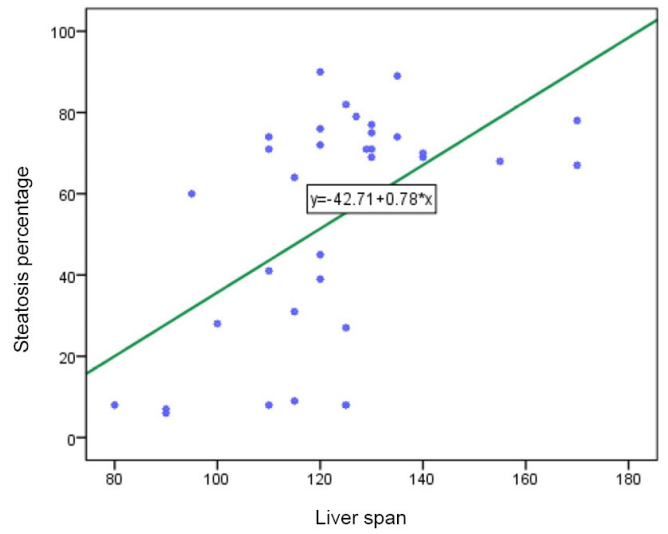

Figure 2. Correlation between liver span and steatosis percentage (Spearman's correlation coefficient $=0.527, P$ value $=0.001$. 
and was not correlated with disease severity and steatosis grades in adolescents with NAFLD. There is limited evidence about DPI in adolescents with NAFLD. In contrast to the findings of this study, Kakkos et al reported that obese adult patients had significantly higher DPI values than healthy volunteers. In addition, they indicated that the grade of fatty liver was inversely associated with DPI (16). In the study by Dugoni et al, DPI was significantly higher in adults with NAFLD and was highly predictive of fatty liver in these patients (27). The differences between our findings with the studies by Kakkos et al (16) and Dugoni et al (27) can be explained by the fact that subjects in these studies were obese and overweight adult patients whereas the patients in our study were adolescents with NAFLD.

One of the important findings of our study was that portal vein parameters in adolescents with NAFLD were significantly lower than those of healthy subjects, since PSV, TAMV, and area of the portal vein were significantly associated with disease severity and steatosis grades. In line with this finding, previous studies focusing on adults suggest that most of the portal vein parameters were found to be lower in patients with fatty liver as compared with controls.

Balasubramanian et al reported that the velocity of the portal flow and the portal vein pulsatility index in patients with fatty liver were significantly lower than those of the controls and were inversely correlated with the severity of fatty liver (28). In addition, some studies have reported similar findings with respect to the velocity of the portal flow and the portal vein pulsatility index $(29,30)$. HABF volume in our study was significantly lower in patients as compared with controls. The mentioned finding was in line with findings of Uzun et al, however, in contrast to findings of Uzun et al, HABF volume was not associated with severity of fatty liver in our study (31). In contrast to our findings, the study by Hizli et al showed that hepatic arterial resistive index in obese children with NAFLD was significantly higher than that of control group (32). This difference may be due to different study samples as the present study addressed adolescents with NAFLD that were a little overweight while the studies by Uzun et al (31) and Hizli et al (32) studied obese children with NAFLD.

Another significant finding of our study was that the liver span of fatty liver was positively correlated with the steatosis percentage. In line with this finding, the study conducted by Balasubramanian et al addressing adults has reported that the liver span of adult patients with NAFLD was significantly higher than that of healthy controls and was correlated with the severity of the fatty liver (28).

\section{Conclusion}

In conclusion, our study revealed that DPI was not different between adolescents with NAFLD and healthy subjects; however, portal vein parameters based on ultrasound were significantly lower in adolescents with NAFLD as compared with healthy subjects. Moreover, the present study indicated that in these patients the percentage of steatosis was significantly related to liver span in adolescents with NAFLD. However, studies with larger sample sizes are warranted to assess the accuracy of DPI and Doppler indices for diagnosis of NAFLD in adolescents.

\section{Limitations of the study}

This study has some limitations. The first limitation of the present study was a relatively small sample size of patients and subsequently a low-number of patients that were categorized according to NAFLD severity and steatosis grades in the sub-group analysis. The mentioned limitation may have reduced the power of statistical tests to detect the differences. Another limitation of the study was potential selection bias. As adolescents in the present study were enrolled from only a single center, findings may not be applicable to a more general population. In addition, transient elastography was used in this study as the standard procedure, and diagnosis of NAFLD was not confirmed by biopsy, while liver biopsy is not a feasible test to pursue the screening purpose.

\section{Authors' contribution}

$M R, H S$, and MF KE were the principal investigators of the study. HS, $M F$, and SHPA were involved in preparing the concept and design. MR and SHPA revisited the manuscript and critically evaluated the intellectual contents. All authors participated in preparing the final draft of the manuscript, revised the manuscript, and critically evaluated the intellectual contents. All authors have read and approved the content of the manuscript and confirmed the accuracy or integrity of any part of the study.

Conflicts of interest

The authors declare that they have no conflict of interest.

Ethical considerations

Ethical issues including plagiarism, data fabrication, and double publication have been completely attended to by the authors.

\section{Funding/Support}

This study was supported by deputy of research and technology of Isfahan University of Medical Sciences, Isfahan, Iran (Grant\# 396754).

\section{References}

1. Angulo P. Nonalcoholic fatty liver disease. $N$ Engl J Med. 2002;346:1221-31. doi: 10.1056/NEJMra011775

2. Sansom SE, Martin J, Adeyemi O, Burke K, Winston C, Markham S, Go B, Huhn G. Steatosis rates by liver biopsy and transient elastography with controlled attenuation parameter in clinical experience of hepatitis $\mathrm{C}$ virus (HCV) and human immunodeficiency virus/HCV coinfection in a large US hepatitis clinic. InOpen Forum Infec Dis. 2019;6(4):ofz099. doi: 10.1093/ofid/ofz099

3. Mann J, Valenti L, Scorletti E, Byrne C, Nobili V. Nonalcoholic Fatty Liver Disease in Children. Semin Liver Dis. 2018;38:113. doi: 10.1055/s-0038-1627456

4. Schwimmer JB, Deutsch R, Rauch JB, Behling C, Newbury 
R, Lavine JE. Obesity, insulin resistance, and other clinicopathological correlates of pediatric nonalcoholic fatty liver disease. Pediatrics. 2003;143:500-5. doi: 10.1067/ S0022-3476(03)00325-1

5. Kimm SY, Obarzanek E. Childhood obesity: a new pandemic of the new millennium. Pediatrics. 2002;110:1003-7. doi: 10.1542/peds.110.5.1003

6. Ruhl CE, Everhart JE. Epidemiology of nonalcoholic fatty liver. Clin Liver Dis. 2004;8:501-19. doi: 10.1016/j.cld.2004.04.008

7. Browning JD, Szczepaniak LS, Dobbins R, Horton JD, Cohen JC, Grundy SM, et al. Prevalence of hepatic steatosis in an urban population in the United States: impact of ethnicity. J Hepatol. 2004;40:1387-95. doi: 10.1002/hep.20466

8. Nobili V, Alisi A, Newton KP, Schwimmer JB. Comparison of the phenotype and approach to pediatric vs adult patients with nonalcoholic fatty liver disease. J Gastroenterol. 2016;150:1798-1810. doi: 10.1053/j.gastro.2016.03.009

9. Suzuki D, Hashimoto E, Kaneda H, Tokushige K, Shiratori K. Liver failure caused by non-alcoholic steatohepatitis in an obese young male. J Gastroenterol Hepatol 2005;20:327-9. doi: 10.1111/j.1440-1746.2005.03724.x

10. Brunt EM, Tiniakos DG. Pathology of steatohepatitis. Best Pract Res Clin Gastroenterol. 2002;16:691-707. doi: 10.1053/ bega.2002.0326.

11. Moran JR, Ghishan FK, Halter SA, Greene HL. Steatohepatitis in obese children: a cause of chronic liver dysfunction. Am J Gastroenterol. 1983;78(6). doi: 10.1111/j.1572-0241.1983. tb01898.x

12. Baldridge AD, Perez-Atayde AR, Graeme-Cook F, Higgins L, Lavine JE. Idiopathic steatohepatitis in childhood: a multicenter retrospective study. Pediatrics. 1995;127:700-4. doi: 10.1016/ S0022-3476(95)70156-7

13. Roberts EA. Pediatric nonalcoholic fatty liver disease (NAFLD): a "growing" problem? J hepatol. 2007;46:1133-42. doi: 10.1016/j.jhep.2007.03.003

14. Roberts EA. Pediatric nonalcoholic fatty liver disease (NAFLD): a "growing" problem? J. Hepatol. 2007;46(6):1133-420. doi: 10.1016/j.jhep.2007.03.003

15. Schwimmer JB, McGreal N, Deutsch R, Finegold MJ, Lavine JE. Influence of gender, race, and ethnicity on suspected fatty liver in obese adolescents. Pediatrics. 2005;115(5):e561-e5. doi: 10.1542/peds.2004-1832

16. Kakkos S, Yarmenitis S, Tsamandas A, Gogos C, Kalfarentzos F. Fatty liver in obesity: relation to Doppler perfusion index measurement of the liver. Scand J Gastroenterol. 2000;35(9):976-80. doi: 10.1080/003655200750023066

17. Mottin CC, Moretto M, Padoin AV, Swarowsky AM, Toneto MG, Glock L, et al. The role of ultrasound in the diagnosis of hepatic steatosis in morbidly obese patients. Obes Surg. 2004;14(5):635-7. doi: 10.1381/096089204323093408.

18. Foucher J, Chanteloup E, Vergniol J, Castera L, Le Bail B, Adhoute $X$, et al. Diagnosis of cirrhosis by transient elastography (FibroScan): a prospective study. Gut. 2006;55(3):403-8. doi: 10.1136/gut.2005.068585

19. De Ledinghen V, Beaugrand M, Kelleher T, Foucher J, Castera L, Ziol M, et al. 87 Prediction of liver fibrosis in non-alcoholic steatohepatitis (NASH): Risk factors and diagnostic potential of liver elasticity using fibroscan. J Hepatol. 2006;44:S39. doi: 10.1016/S0168-8278(06)80088-7

20. Preiss D, Sattar N. Non-alcoholic fatty liver disease: an overview of prevalence, diagnosis, pathogenesis and treatment considerations. Clin Sci. 2008;115):141-50. doi: 10.1042/ CS20070402

21. Myers RP, Elkashab M, Ma M, Crotty P, Pomier-Layrargues G. Transient elastography for the noninvasive assessment of liver fibrosis: a multicentre Canadian study. Canadian J Gastroenterol Hepatol. 2010;24:661-70. doi: 10.1155/2010/153986

22. Friedrich-Rust M, Ong MF, Martens S, Sarrazin C, Bojunga J, Zeuzem S, et al. Performance of transient elastography for the staging of liver fibrosis: a meta-analysis. J Gastroenterol. 2008;134:960-74. doi: 10.1053/j.gastro.2008.01.034

23. Castéra L, Foucher J, Bernard PH, Carvalho F, Allaix D, Merrouche $W$, et al. Pitfalls of liver stiffness measurement: a 5-year prospective study of 13,369 examinations. J Hepatol. 2010;51:828-35. doi: 10.1002/hep.23425

24. Foucher J, Castéra L, Bernard P-H, Adhoute X, Laharie D, Bertet J, et al. Prevalence and factors associated with failure of liver stiffness measurement using FibroScan in a prospective study of 2114 examinations. Eur J Gastroenterol Hepatol. 2006;18:411-2. doi: 10.1097/00042737-200604000-00015

25. Myers RP, Pomier-Layrargues G, Kirsch R, Pollett A, DuarteRojo A, Wong D, et al. Feasibility and diagnostic performance of the FibroScan XL probe for liver stiffness measurement in overweight and obese patients. J Hepatol. 2012;55:199-208. doi: 10.1002/hep.24624

26. Leen E, Goldberg JA, Angerson WJ, McArdle CS. Potential role of Doppler perfusion index in selection of patients with colorectal cancer for adjuvant chemotherapy. Lancet. 2000;355:34-7. doi: 10.1016/S0140-6736(99)06322-9

27. Dugoni M, Miglioli L, Borelli L, Anderlini R ,Bedogni G, Mariano $\mathrm{M}$, et al. Doppler perfusion index (DPI) and homa are highly predictive of fatty liver in patients with NAFLD. Dig Liver Dis. 2008;40:A39. doi: 10.1016/j.dld.2007.12.097

28. Balasubramanian P, Boopathy V, Govindasamy E, Basavaiya V. Assessment of Portal Venous and Hepatic Artery Haemodynamic Variation in Non-Alcoholic Fatty Liver Disease Patients. J Clin Diagn Res. 2016:10:TC07-TC10. doi: 10.7860/ JCDR/2016/20697.8267

29. Erdogmus B, Tamer A, Buyukkaya R, Yazici B, Buyukkaya $A$, Korkut E, et al. Portal vein haemodynamics in patients with non-alcoholic fatty liver disease. Tohoku J Exp Med. 2008;215:89-93. doi: 10.1620/tjem.215.89

30. Balci A, Karazincir S, Sumbas H, Oter Y, Egilmez E, Inandi T. Effects of diffuse fatty infiltration of the liver on portal vein flow haemodynamics. J Clin Ultrasound. 2008;36:134-40. doi: 10.1002/jcu.20440

31. Uzun H, Yazici B, Erdogmus B, Kocabay K, Buyukkaya R, Buyukkaya A, et al. Doppler waveforms of the hepatic veins in children with diffuse fatty infiltration of the liver. Eur J Radiol. 2009;71:552-6. doi: 10.1016/j.ejrad.2008.06.004

32. Hizli S, Koçyigit A, Arslan N, et al. Hepatic artery resistance in children with obesity and fatty liver. Indian J Pediatr. 2010;77: 407-411. doi: 10.1007/s12098-010-0045-x 